\title{
On Deflection of Potentially Dangerous Asteroids
}

\author{
Josh Fixelle and Mikhail Kagan* \\ Department of Science and Engineering, Pennsylvania State University, Abington \\ *Mentor:mak411@psu.edu
}

\begin{abstract}
As has been widely discussed recently, our planet may become a target for asteroids. We consider several scenarios proposed to prevent asteroid collisions with Earth. The asteroid 99942 Apophis is considered as a typical representative. Among others, the recent "gravitational tractor" scenario ${ }^{2}$ is discussed. For a simplistic toy-model we obtain estimates for both the mass of the tractor and the amount of fuel required to tow a potentially dangerous asteroid off-course so as to avoid a collision with the Earth. In addition, we analyze two more scenarios titled "sling-shot" and "bumping", and comment on their relative efficiency compared to the "towing" scenario. Based on the analysis, the bumping scenario looks most promising.
\end{abstract}

\section{KEYWORDS}

Near-Earth objects, Gravitational Tractor, Asteroid 99942 Apophis

\section{INTRODUCTION}

After watching a presentation on the possibility of asteroids colliding with the planet Earth, a thought occurred: this may be reality in the near future. In fact, due to one specific asteroid (99942 Apophis), this idea may be even more of a reality than first thought. Situations such as an asteroid impact have been depicted in disaster films since the late twentieth century, all with the same solution - to destroy the asteroid with high yield explosives. There is however an issue with the use of explosives: an asteroid has an original quantity of energy (kinetic, potential, etc.). If the asteroid were to be split into smaller pieces (as an explosion would), then the only change occurring is that the overall energy is then concentrated throughout several pieces instead of just one, and thus after collision the energy is spread over several impact locations as opposed to concentrated at one. Another consideration is that of aerodynamic heating. Most small space born objects collide with the Earth's atmosphere at high velocities, which in turn generates friction between the object and the atmosphere - this friction will tend to disintegrate most of the materials that enter the Earth's atmosphere.

With that said, the small asteroid pieces - resulting from the explosive device - may or may not disintegrate (depending on their volume) upon entry. This aerodynamic heating may result in anything from minimal heating of the Earth's atmosphere, to a more notable heating - which could in turn create meteorological changes throughout the planet. One may also consider the fact that small asteroid pieces - the ones that do not disintegrate upon reentry - may still cause significant damage (consider if one were to impact in the center of a large city). When the asteroid is broken up, the initial energy remains the same (with the addition of the energy from the explosion), and thus will still be transferred to the Earth upon collision (over a larger area, and in multiple forms) - the exception to this would be considering a transfer of momentum to pieces due to the explosion, giving them a trajectory that does not collide with the Earth. These non-colliding pieces however, would be insignificant when compared to the amount that would in fact still collide with the Earth. With that said, we realized that as a planet we currently have no viable means to "defend" against a possible asteroid collision.

\section{AJUR volume 12 | Issue 1 | August 2014}




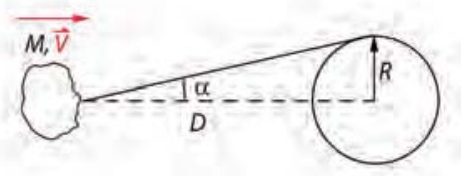

Figure 1. Asteroid of mass $M$ moving at speed $V$ is approaching the Earth. A safe scenario must ensure that the asteroid will be deflected sideways by the minimum angle $\alpha=\frac{R}{D}$, where $R$ is the radius of the Earth, and $D$ is the intercept distance.

A typical simplistic problem can be formulated as follows. Consider a potentially dangerous asteroid on a collision course with the Earth, as illustrated in Fig.(1). The asteroid has mass $M$, velocity $\vec{V}$ and, in the worst case scenario, zero impact parameter, that is its extended trajectory passes through the center of the Earth. The latter implies that the asteroid should be deflected sideways by at least one radius of the Earth, $R$. Assuming that the asteroid is detected (and gets acted upon) at distance $D$ from the Earth, its trajectory must be deflected by a small angle $\alpha=\frac{R}{D}$. For the reader's convenience we summarize all the relevant numerical data in the table below, taking the 99942 Apophis as a typical representative. Footnote 1

\begin{tabular}{|l|l|}
\hline Physical quantity & Numerical Value \\
\hline Radius of the Earth, $R$ & $6.37 \cdot 10^{6} \mathrm{~m}$ \\
\hline Intercept distance, $D$ & $2.55 \cdot 10^{11} \mathrm{~m}$ \\
\hline Minimum deflection angle, $\alpha=\frac{R}{D}$ & $2.50 \cdot 10^{-5}$ \\
\hline Speed of the asteroid, $V$ & $3.07 \cdot 10^{4} \frac{\mathrm{m}}{\mathrm{s}}$ \\
\hline Travel time, $t=\frac{D}{V}$ & $8.31 \cdot 10^{6} \mathrm{~s} \approx 0.263$ years \\
\hline Mass of the asteroid, $M$ & $2.10 \cdot 10^{10} \mathrm{~kg}$ \\
\hline Gravitational constant, $G$ & $6.67 \cdot 10^{-11} \frac{\mathrm{m}^{3}}{\mathrm{~kg} \cdot \mathrm{s}^{2}}$ \\
\hline
\end{tabular}

Table 1. Typical numerical data relevant for a collision problem. The mass and speed of a typical asteroid correspond to those of 99942 Apophis.

Provided that the potentially dangerous asteroid is detected early enough and is intercepted sufficiently far away from the Earth, it needs to be deflected by just a tiny angle $\alpha \sim 10^{-5}$ (see Table 1) to prevent collision. This fact gave rise to a promising idea that there should a viable method of deflecting the asteroid on a collision course with the Earth (rather than using high yield explosives), by means that are available with current day technology. ${ }^{1}$

Footnote 1 In reality, 99942 Apophis follows a nearly circular orbit around the Sun and appears in a close proximity of the Earth once every seven years. In our toy model, however, we assume the potentially dangerous asteroid to be on a straight collision course with the Earth, and take the mass and speed of 99942 Apophis just for sake of estimate. In addition, we consider the problem in the reference frame associated with the Earth.

\section{AJUR volume 12 | Issue 1 | August 2014}




\section{SCENARIO 1. DEFLECTION BY GRAVITATIONAL PULL}

It was recently suggested ${ }^{2}$ that by flying a small spacecraft ('gravitational tractor') alongside an asteroid, the gravitational pull created from the spacecraft could in fact alter the trajectory of the asteroid, avoiding impact entirely.

In Fig. 2, a "gravitational towing" scenario is illustrated. A spacecraft is hovering near the asteroid at a constant distance $d$ (as measured from the center of the asteroid), thus creating a constant force of gravity acting on the asteroid in a sideways direction. The spacecraft's thrusters provide a force exactly needed to prevent the spacecraft from falling onto the asteroid. For simplicity, we neglect the pressure of the fuel exhaust on the surface of the asteroid. Below we investigate this scenario and provide some numerical estimates.

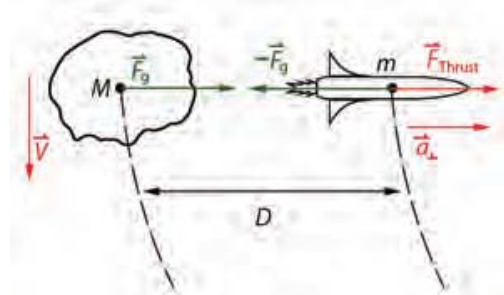

Figure 2. Scenario 1. Asteroid is being deflected by the gravitational pull exerted by a spacecraft of mass $m$ flying alongside at a constant distance $d$ away from the asteroid.

\subsection{ESTIMATION OF THE SPACECRAFT MASS}

The gravitational force on the asteroid provides acceleration

$$
a=\frac{G m}{d^{2}},
$$

Equation 1.

which results in a sideways displacement

$$
\Delta x=\frac{1}{2} a t^{2} .
$$

Here $t=\frac{D}{V}$ is the time it takes for the asteroid to reach the Earth (see Table 1). Setting the deflection equal to the radius of the Earth gives us an estimate for the minimum mass of the spacecraft

$$
m=\frac{2 R d^{2} V^{2}}{G D^{2}} \text {. }
$$

Equation 2.

Substituting the values from Table 1 and assuming that $d \approx 175 \mathrm{~m}$, we obtain

$m \approx 8.478 \cdot 10^{7} \mathrm{~kg}$.

For comparison, the mass of the 99942 Apophis asteroid is $2.1 \cdot 10^{10} \mathrm{~kg}$, whereas the mass of the NASA space shuttle is $2.03 \cdot 10^{6} \mathrm{~kg}$. With that said, the mass required to deflect an asteroid with the velocity of the 99942 Apophis asteroid with an impact parameter of zero is more than that of the NASA space shuttle. Thus the idea so far is implausible, as the mass required is around that of 37 NASA space shuttles (not including the fuel required). For the sake of completion, we will still continue the analysis. 


\subsection{ESTIMATION OF THE REQUIRED AMOUNT OF FUEL}

The next step in investigating the gravitational towing scenario is to determine if the amount of fuel (mass) required to accomplish this mission is remotely plausible. The most prevalent fuel requirement is that of the necessity to keep the spacecraft from colliding with the asteroid due to gravitational attraction. There is also the fuel requirement to place the spacecraft in orbit, and to send the spacecraft the distance required to intercept the asteroid, however for now those requirements can be assumed negligible. Since the separation between the asteroid and the spacecraft remains constant, in the reference frame associated with the Earth, the spacecraft is moving with the same acceleration as the asteroid. Therefore the thrust generated by the engines should exceed the gravitational force

$$
F=\frac{G M m}{d^{2}}
$$

by $m a$, where $a$ is the acceleration from Eq.(1). In other words, the engines should provide the force

$$
F^{\prime}=\frac{G(m+M) m}{d^{2}} \approx \frac{G M m}{d^{2}}
$$

The latter is given by

$$
F^{\prime}=v_{\text {rel }}\left|\frac{d m}{d t}\right|,
$$

where $v_{\text {rel }}$ is the exhaust speed of the fuel, and $\left|\frac{d m}{d t}\right|$ is the mass rate. Using again the time from Table 1, we obtain an estimate for the total amount of fuel needed to tow the asteroid off-course

$$
\begin{aligned}
\Delta m & =\left|\frac{d m}{d t}\right| \frac{D}{V} \\
& =\frac{G M m}{d^{2} v_{r e l}} \frac{D}{V} .
\end{aligned}
$$

Equation 3.

Assuming the minimum mass found in Eq.(2), the mass of the required fuel can be written as

$$
\Delta m=2 \alpha M \frac{V}{v_{\text {rel }}} \text {. }
$$

Equation 4.

To estimate the amount of fuel we take the exhaust speed for the J-2s engine,

$v_{\text {rel }}=4.28 \cdot 10^{3} \mathrm{~m} / \mathrm{s}$, which yields

$\Delta m \approx 7.53 \cdot 10^{6} \mathrm{~kg}$.

The latter is approximately four times that of the mass of the NASA space shuttle, and still less than the mass of the spacecraft itself. In other words, this idea is plausible if the mass of the spacecraft was reasonable, however due to the enormous mass of the spacecraft and the large mass of the fuel required to maintain the distance of separation between the spacecraft and asteroid, the idea is not plausible.

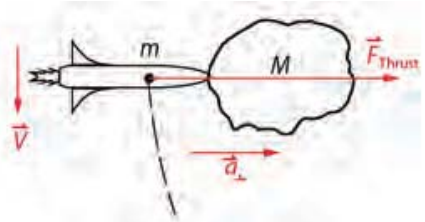

Figure 3. "Pushing scenario". Asteroid is being deflected off-course by the engines a spacecraft landed on its surface. 


\subsection{COMPARISON WITH THE "PUSHING SCENARIO"}

The towing scenario discussed above was suggested, ${ }^{2}$ as a less technically demanding way of deflecting asteroid off its collision course. The advantage being is that the tractor is insensitive to the structure of the asteroid but its mass. Moreover, it avoids landing on the surface of the asteroid which is one of the most challenging cosmic tasks. In addition, even if the landing has been successful, rotation of the asteroid makes it extremely difficult to direct the thrust, as well as merely to control the (unmanned) spacecraft, as it might get out of the line of sight.

Suppose, however, that the challenges associated with landing the spacecraft on the asteroid and navigating it have been successfully overcome (see Figure 4 ). What would be the required specifications of the spacecraft and the amount fuel to deflect the asteroid off-course by the same amount as before? We start by noting that now the spacecraft and the asteroid constitute one system, thus subjects to one common force provided by the spacecraft's engines. Clearly, only the combined mass, $M+m$, now matters, in which the mass of the spacecraft can be neglected, as $m \ll M$. Moreover, since the relative speed of the exhausted fuel $v_{r e l}$ is much greater than the sideways velocity of the system, the total sideways impulse provided by the engines can estimated as

$$
\Delta P_{\perp} \approx \Delta m v_{\text {rel }} \text {. }
$$

On the other hand, a successful deflection would require a sideways momentum of the asteroid of approximately

$$
\Delta P_{\perp}=\alpha P_{\|} \equiv \alpha M V .
$$

The latter leads to the following estimate for the mass of the fuel

$$
\Delta m=\alpha M \frac{V}{v_{r l}} . \quad \text { Equation } 5 .
$$

This expression gives a generic estimate for the amount of the required fuel regardless the details of a particular "towing" or "pushing" scenario. It is easy to see that the above result has the same order of magnitude as in Eq. (4).

To clarify the seeming discrepancy in the numerical prefactor between Eqs.(4) and (5), we should note that providing the asteroid with a transversal momentum $P_{\perp}=\alpha P_{\|}$is not yet sufficient to deflect it by an Earth radius $R$, unless the impulse is impeded over a very short period of time (much shorter than the travel time) in the beginning of the deflection phase. A scenario that realizes this idea is discussed in the next section.

For a long-term "pushing" or "towing", one needs to approach the problem along the lines of the previous subsection by considering the (constant) sideways acceleration as in Eq.(1). It is then easy to see that the corresponding thrust force should be exactly the same as that required in the previous subsection to prevent the spacecraft from falling onto the asteroid. Indeed, when computing the total external (towing) force acting upon the spacecraft-asteroid system (see Fig. 2), the two gravitational forces mutually cancel yielding the same resultant force.

We thus arrive at the conclusion that the only advantage of the "pushing" scenario is that it may work with a lighter spacecraft, as its mass would be irrelevant. It is, however, impossible to safe fuel. 


\section{SCENARIO 2. SLING-SHOT DEFLECTION.}

The main idea behind this scenario is to "sling-shot" a spacecraft over the asteroid. If the spacecraft passes close enough to the asteroid (i.e. the spacecraft's impact parameter $\rho$ is sufficiently small) due to gravitational attraction the asteroid will also be deflected off its collision course. Provided that the asteroid needs to be deflected a very small angle $\alpha \sim 10^{-5}$, the scenario may turn out to be quite realistic.

The "sling-shot" scenario is illustrated in Fig.4. On the left is the scattering as viewed in the reference frame associated with the asteroid (A-frame). The spacecraft approaches from the bottom with initial speed $v_{\infty}$ and impact parameter $\rho$. It then undergoes elastic Coulomb scattering by angle $\theta$, so that the final speed of the spacecraft far away from the asteroid is also equal $v_{\infty}$. The same process as viewed in the laboratory frame (L-frame), e.g. heliocentric frame, is depicted on the right of Fig.4. The asteroid scatters by a small angle $\alpha$ and receives a transversal velocity $\vec{V}_{\perp}$ and momentum

$\Delta P_{\perp}=\alpha P_{\|}=\alpha M V$

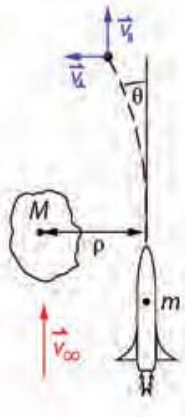

Equation 6.

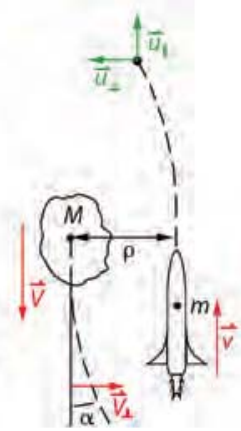

Figure 4. Scenario 2. The spacecraft is "sling-shot" over the asteroid so as to deflect it by gravitational attraction. The left image is in the frame of the asteroid (A-frame), and the right image is in a laboratory frame, e.g. heliocentric frame (L-frame).

which is equal to the transversal momentum of the spacecraft. Rigorously speaking, the A-frame is not inertial. However, given that typical changes in the asteroid's velocity, hence its acceleration, are small (of relative order of $\alpha$ ) compared to the typical speeds, the A-frame can be considered inertial with the required accuracy. Furthermore, up to a negligible amount, the transversal momentum of the spacecraft after collision is the same in both frames and equals that in Eq.(6).

In the light of this, a successful sling-shot scenario would require maximization of the transversal impulse received by the spacecraft during the scattering. Given fixed $v_{\infty}$ and $\rho$, the maximum transversal impulse $\Delta p_{\perp}$ can be attained if the spacecraft is launched at initial angle $\theta / 2$ to the right from the vertical. In that case, it will scatter at angle $\theta / 2$ to the left from the vertical, resulting in

$$
\Delta p_{\perp}=m \Delta v_{\perp}=m v_{\infty}\left(2 \sin \left(\frac{\theta}{2}\right)\right)
$$

It is easy to see that the situation depicted in Fig. 4 would correspond to a factor of $\sin \theta$ instead of the one in the parenthesis and lead to a smaller transversal impulse. Footnote 2

Footnote 2 Clearly, $\frac{\sin \theta}{2 \sin (\theta / 2)}=\cos (\theta / 2)<1$. 
The scattering angle is given by ${ }^{3}$

$$
\tan \left(\frac{\theta}{2}\right)=\frac{G M}{\rho v_{\infty}^{2}} \quad \text { Equation } 8 .
$$

Using Eq.(7) and the inequality $\sin (\theta / 2)<\tan (\theta / 2)$, we obtain the following upper bound on the transversal impulse

$$
\Delta p_{\perp}<2 \frac{G m M}{\rho v_{\infty}}=m \frac{v_{c s c}^{2}}{v_{\infty}},
$$

where $v_{e s c}=\sqrt{\frac{2 G M}{\rho}}$ is the escape velocity from the asteroid. Obviously, $v_{\infty}$ must be greater than vesc, which implies that

$$
\Delta p_{\perp}<m v_{e s c}
$$

For the asteroid at hand, the mass and the impact parameter are of order $M \sim 10^{10} \mathrm{~kg}$ and $\rho \sim 100 \mathrm{~m}$ respectively (see Table 1), which corresponds to an escape velocity of order of $0.1 \mathrm{~m} / \mathrm{s}$. Clearly, the bound above makes it impossible for the spacecraft to have a sufficient change in transversal momentum of order of $\alpha M V \sim m V$, with the required speed $V \sim 10^{4} \mathrm{~m} / \mathrm{s}$. Let us remark on this result from a different perspective. As estimated above, the minimum transversal impulse of the asteroid should be such that the spacecraft's sideways speed is of order of the asteroid's speed $V \sim 10^{4} \mathrm{~m} / \mathrm{s}$. While this is a realistic value for the spacecraft's speed, the transfer of the corresponding sideways momentum to the asteroid requires strong enough interaction between the bodies. In other words, the deflection would be successful if the spacecraft simply (inelastically) hit the asteroid sideways and transferred the needed impulse through contact forces. As the analysis above shows, the transfer cannot be realized at a sufficient rate if the interaction is purely gravitational. We investigate the "bumping" possibility in the next section.

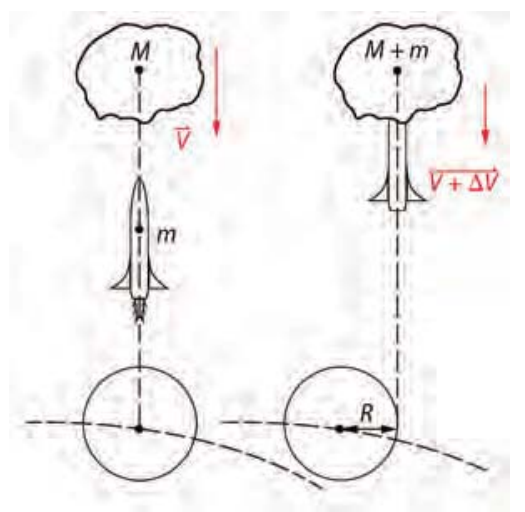

Figure 5. Scenario 3. Asteroid is moving in the ecliptic plane towards the Sun so that it would reach the Earth's orbit exactly when the Earth is on the line Sun-Asteroid. 


\section{SCENARIO 3. "BUMPING"}

Another promising collision avoidance possibility is associated with slowing the asteroid down rather than deflecting it off-course. From now on we consider the heliocentric reference frame and suppose, for simplicity, that the asteroid is approaching the orbit of the Earth along a radial direction (see Fig. 5). Initially, the asteroid is at distance $D$ away from the Earth's orbit and is moving with velocity $V$ towards the Sun. If no action is undertaken, the asteroid will collide with the Earth at the intersection of the orbit with the line Sun-Asteroid.

Unlike the previous sections, timing is critical in this scenario. If the asteroid crosses the Earth's orbit later (or earlier), the collision would be avoided. In fact, it would be sufficient if the asteroid is delayed by an amount of time, $t_{\perp}$, such that the Earth would have traveled at least one its radius, $R$. Specifically the minimum delay is given by

$$
t_{\perp}=\frac{R}{V_{\perp}}
$$

where $V_{\perp}$ is the Earth's orbital speed. It is easy to see that the delay is much less than the travel time. Indeed,

$$
\frac{t_{\perp}}{t}=\frac{R}{D} \frac{V}{V_{\perp}} \approx \alpha \ll 1 .
$$

In the last approximation we have used the fact that $V_{\perp} \approx V$.

Relative smallness of the delay time allows to simplify the further analysis. In particular, delaying the asteroid by $t_{\perp}$ requires slowing it down by $\Delta V$ such that

$$
\frac{\Delta V}{V}=\frac{t_{\perp}}{t}=\alpha \frac{V}{V_{\perp}} .
$$

The slowing down can be realized by using the "Bumping" scenario, that is by placing a relatively small obstacle of mass $m$ on the asteroid's way. After the collision with the bump, the fractional decrease in the asteroid's speed would be equal to $m / M$. Therefore, the mass of the obstacle, should constitute the following fraction of the asteroid's mass:

$$
\frac{m}{M}=\frac{\Delta V}{V}=\frac{t_{\perp}}{t}=\alpha \frac{V}{V_{\perp}} . \quad \text { Equation } 9 .
$$

For Apophis, the minimum required mass of the bump would be

$$
m \approx \alpha M=5.25 \cdot 10^{5} \mathrm{~kg} \text {, }
$$

which is much smaller than that in the previous scenarios. 


\section{DISCUSSION}

We have considered several possible scenarios for deflecting a potentially dangerous asteroids off their collision course with the Earth and estimated the minimum required mass of the spacecraft, as well as the amount of fuel needed during the deflecting stage. Remarkably, the mass of the fuel (when applicable) and the mass of the "bump" in the bumping scenario turned out to be of the same order of magnitude given by

$$
m \approx \alpha M \frac{V}{V_{\perp}},
$$

where $\alpha$ is the deflection angle, $M$ and $V$ are the mass and speed of the asteroid. The denominator, $V_{\perp}$, is either the exhaust speed of the fuel (in the scenarios involving engines) or the sideways (orbital) speed of the Earth. Typically, the mass of the spacecraft (in the corresponding scenarios) is by an order of magnitude greater than that of the fuel. In the light of this, the bumping scenario looks much simpler and cheaper, as it only requires placing the bump at the "appropriate" location. Although that might be a non-trivial task, it still avoids many complications associated with spacecraft maneuvering. Although the gravitational towing scenario may be efficient in deflecting an asteroid away from a "key-hole", it is less so when a deflection by a radius of the Earth is required.

In order to minimize the mass of the fuel in the engines-based scenarios, one should aim at a greater $V_{\perp}$. In that sense, engines with greater $v_{r e l}$, but not necessarily with greater mass rate $\frac{d m}{d t}$, would be preferred. For instance the VASIMR engine currently being developed by Ad Astra Rockets has a propellant velocity measured as high as $2 \cdot 10^{4} \mathrm{~m} / \mathrm{s}$ and a mass rate of $0.07 \mathrm{~g} / \mathrm{s} .{ }^{4}$ Granted, with these current specifications, multiple engines would be needed, however, they are close to achieving what would be needed.

Comparative analysis of the "towing" and "pushing" scenarios (see Lu and Love ${ }^{2}$ and Section 1.3) indicates that, in general, towing is more promising. Pushing, however may be more efficient in case of a weak gravitational pull. For there is no limit on the pushing force, while the towing force (force of gravity between the tractor and the asteroid) should not be exceeded by $F_{\text {thrust }}$, so as to not $v=$ break the "tow-line". We note, however, that in the two examples discussed above, $F_{G} \approx F_{\text {thrust }} \approx$ $100 \div 1000$ (newtons). A few additional remarks are in order. Lu and Love ${ }^{2}$ took into consideration the fact that the thrust must not interact with the asteroid's surface (i.e. the thrust must be vectored so that it does not exert a force on the asteroid's surface, thus ensuring that the distance of separation does not increase, or the force of gravity between the two bodies is not canceled out by the thrust). We however did not take this into consideration, which would mean that a slightly greater thrust would be needed, thus more fuel must be used. In addition, we did not consider the escape velocity of the asteroid. This further limits the type of thruster which can be used, as if the impulse of the thrusters is too great, the spacecraft will "escape" from the gravitational field of the asteroid, and thus nullify the Gravitational Tractor.

There is another issue with the scenario proposed by Lu and Love. ${ }^{2}$ The scenario requires an extremely long period of time for the tractor to actually be practical (i.e. the asteroid must be intercepted at a long distance, a distance which will most likely not be seen in respect to our solar system, and with current day technology, would not be feasible to reach). The scenario will mostly only work for a specific example such as that of the 99942 Apophis asteroid, and thus has limited application.

Another possible issue with this is the distance of separation. If we take into consideration the 99942 Apophis asteroid - with an estimated length of 320 meters - a distance of separation of 175 meters may not be sufficient. Consider the fact that most asteroids have some spin to them, and that the distance of separation is measured from the center of mass of both bodies. This means that depending upon the spin of the asteroid, the spacecraft at a distance of separation of 175 meters may be within the "sweep area" (the total volume of space - in reference to the asteroids center of mass - occupied by the asteroid due to spin as $t \rightarrow \infty$ ), and thus may result in a collision. To correct 
for this, a larger mass of the spacecraft would be needed, and consequently more fuel, stretching the plausibility of the scenario even further.

Some other deflection ideas to test include the following: One of JPL's suggestions is to simply land a rocket on the surface of the asteroid, and fire its rockets, so that it changed the perpendicular momentum enough to divert it from impacting earth. This along with other "momentum" ideas could be theoretically tested. Besides direct contact, if for example a few massive crafts were fired at the side of the asteroid, that could change the perpendicular momentum. Or, if a high yield explosive is detonated next to the asteroid (not in it), it may transfer enough momentum to the asteroid to deflect it sufficiently. Perhaps consider the idea of the gravitational tractor, and the slingshot effect in terms of the electromagnetic force instead of the gravitational force. If the asteroid was magnetized, and a spacecraft with a super electromagnet was flown with a parallel trajectory, it could attract the asteroid, and they could "meet" (collide), at some point where the impact parameter of the asteroid is now $R$. Also, if the asteroid could be magnetized, the same example as just stated could be used, except instead of destroying the spacecraft, just use it to alter the asteroid's perpendicular momentum more, and perhaps be more efficient. Those two ideas are inspired by the fact that, on general grounds, it is easier to arrange for a stronger electromagnetic interaction than the gravitational one.

\section{ACKNOWLEDGEMENTS}

We thank Dr. Ann Schmiedekamp for helpful discussions and extremely useful information about potentially dangerous asteroids, Dr. Carl Schmiedekamp for pointing out the comprehensive review in Yeomans, ${ }^{1}$ and Dr. Moylan for the discussions on Coulomb scattering. The work of J.F. was generously supported by the ACURA fund of Penn State Abington.

\section{REFERENCES}

[1] Yeomans, D. (2009) Deflecting a Hazardous Near-Earth Object, In Proceedings 1st IAA Planetary Defense Conference: Protecting Earth from Asteroids, Granada, Spain.

[2] Lu, E. T., and Love, S. G. (2005) Gravitational tractor for towing asteroids, Nature 438, 177-178.

[3] Landau, L., and Lifshitz, E. (1976) Theoretical Mechanics, 3rd ed., Butterworth-Heinemann.

[4] Squirel, J. (2008) VASIMR Performance Measurements at Powers Exceeding $50 \mathrm{~kW}$ and Lunar Robotic Mission Applications, In International Interdisciplinary Symposium on Gaseous and Liquid Plasmas, Akiu/Sendai, Japan.

\section{ABOUT THE STUDENT AUTHOR}

Joshua Fixelle graduated from the Pennsylvania State University in December, 2013 with BS degrees in Astronomy \& Astrophysics and Engineering Science \& Mechanics (minors in Mathematics and Physics). He is currently living in Evanston, IL, while attending Northwestern University pursuing his Doctorate in Astrophysics (PhD).

\section{PRESS SUMMARY}

As has been widely discussed recently, our planet may become a target for asteroids (the asteroid 99942 Apophis is considered as a typical representative). We considered several scenarios proposed to prevent asteroid collisions with Earth, including the "gravitational tractor", "sling-shot", and "bumping" scenarios. We analyzed and compared the scenarios in regards to their relative efficiency compared to the "gravitational tractor", and concluded that the bumping scenario looks most promising. 